\title{
Beam breakup instability suppression in multicell superconducting rf guns
}

\author{
V. Volkov* \\ BINP, Novosibirsk, Russia
}

J. Knobloch and A. Matveenko

Helmholtz-Zentrum, Berlin, Germany

(Received 28 November 2009; revised manuscript received 28 November 2010; published 19 May 2011)

\begin{abstract}
In this paper, we provide an analytical description for transverse coupling impedances with dipole high order modes (HOMs) and beam breakup (BBU) instability suppression of relativistic, high-current beams undergoing strong acceleration, such as those typically produced by rf photoinjectors. The model adopted is based on the accepted theory of coupling impedances extended to the case of beams characterized by a fast transition due to strong acceleration, from the nonrelativistic to the relativistic regime in which a bunch trajectory may not be rigidly directed parallel to the axis of the electric field. The trajectory oscillations in a dipole HOM field in the transverse plane are effective in perturbing the bunch energy, which causes an increase of the coupling impedance up to the BBU instability. This BBU instability analysis is based on equations obtained by Volkov [Phys. Rev. ST Accel. Beams 12, 011301 (2009)] in which external focusing due to both the applied transverse electric (TE) HOM and the accelerating fundamental mode as well as dipole HOM damping by external loads are investigated. This analysis is valid in the limit in which the weak nonlinearity of applied fields near the axis may be neglected. The solution suggests a means of enhancing the BBU threshold current. The possibility of obtaining BBU instability suppression in a long, integrated photoinjector and linac structure, such as the superconducting rf source at Forschungs Zentrum Dresden (FZD), are numerically examined. New designs of photoinjectors that provide BBU instability suppression of all dipole HOMs to enhance the threshold currents are suggested.
\end{abstract}

DOI: 10.1103/PhysRevSTAB.14.054202

PACS numbers: 29.27.Bd, 85.25.-j, 41.85.Ja, 29.20.Ej

\section{INTRODUCTION}

Third-generation synchrotron radiation sources in the UV and x-ray spectral region have become available to a wide scientific community and have led to a huge number of applications in different scientific fields. Light sources based on the energy recovery linac (ERL) principle now bear the promise of providing very short pulses at both a high average brightness and flux for many users simultaneously. World-wide, several ERLs are already in operation or are being proposed [1,2]. One of them is the ERL feasibility study BERLinPro that was proposed by the Helmholtz-Zentrum Berlin. Among the most challenging parameters of BERLinPro is the average beam current of $100 \mathrm{~mA}$. The plans call for the development of a superconducting photoinjector to supply this beam, and prototyping is already under way [3].

The beam breakup (BBU) instability analysis of superconducting, multicell cavities in Ref. [4] is extended here to an FZD-like, multicell rf gun [5], as shown in Fig. 1.

\footnotetext{
*V.N.Volkov@inp.nsk.su
}

Published by the American Physical Society under the terms of the Creative Commons Attribution 3.0 License. Further distribution of this work must maintain attribution to the author(s) and the published article's title, journal citation, and DOI.
This gun consists of a $3 \frac{1}{2}$-cell, superconducting system $(1.3 \mathrm{GHz})$. In addition to the usual accelerating mode, the $\mathrm{TE}_{021}$ mode provides a focusing field for the electrons that can also be excited.

First, we analyze the features of BBU instabilities at high-current beams in such injectors. The analytical dependence of $\mathrm{BBU}$ onset for dipole high order modes (HOMs) is obtained, and a table of calculated impedances for the considered rf gun is presented. Next, we analyze the characteristics of trapped dipole modes. It is shown that their amplitudes and quality factors can change significantly when the fundamental mode of the cavity is tuned to the master clock. We then use the calculated HOMs to estimate the threshold current for instabilities. Because the

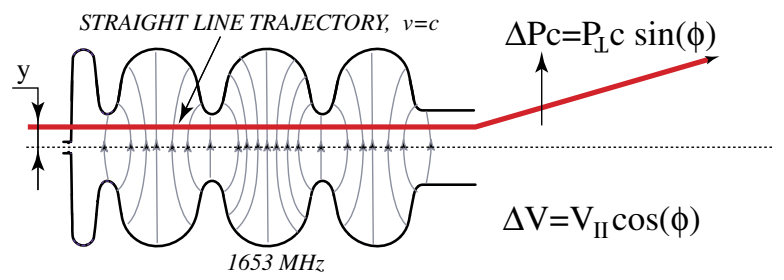

FIG. 1. Beam trajectory (red) conventionally used to calculate the coupling impedances. The electric field lines of the vertically polarized dipole mode $\mathrm{TE}_{111}$ of 0 type with $1653-\mathrm{MHz}$ frequency are depicted. 
impedance is trajectory dependent, the focusing of the TE mode can be used to suppress some instabilities, and its impact is examined. This allows for a new design of an $\mathrm{rf}$ photoinjector able to suppress BBU instability of all dipole HOMs. Finally, we present an option to damp other HOMs using coaxial beam pipe couplers to dramatically increase the threshold currents. New designs of photoinjectors that incorporate these features are suggested.

\section{DERIVATION OF BBU INSTABILITY FEATURES}

In the following discussion, we treat the beam as a series of pointlike bunches entering the cavity at regular intervals. The current thus is a series of delta functions with defined charges. In the frequency domain, the current has an infinite series of Fourier harmonics, all with an amplitude equal to twice the average current $\left(I_{i}=2 I\right)$. Because we measure the average current, we use the Ohm's law formulas for DC current and double the harmonic impedances $\left(R=2 R_{i}\right)$ to keep the voltages and powers unchanged, i.e.,

$$
\begin{gathered}
V_{i}=I_{i} R_{i}=2 I R / 2=I R=V \\
P_{i}=V_{i}^{2} / 2 R_{i}=V^{2} / R=P .
\end{gathered}
$$

\section{A. Brief review of the accepted theory of coupling impedances}

In the paper, a vertically polarized dipole field $\left(E_{y}, B_{x}\right)$ is assumed, and the dipole field strength $B$ is referred to as a maximum dipole magnetic field on an axis, i.e., $B=\max \left(B_{x}\right)$. The excitation of dipole HOMs by the beam current $(I)$ is characterized by longitudinal $\left(R_{I I} \equiv R_{1}\right)$ and transverse $\left(R_{\perp}\right)$ coupling impedances:

$$
\begin{gathered}
V_{I I} \equiv V_{1}=I \cdot R_{1} \\
P_{\perp} c=I \cdot R_{\perp} .
\end{gathered}
$$

Both the induced longitudinal voltage and longitudinal coupling impedance of the accepted theory are denoted here by $V_{1}$ and $R_{1}$, respectively, but in reality, each of them is a constituent part of three-component equations denoted below by $V_{I I}$ and $R_{I I}$.

In the steady state, the excited rf power is equal to the power dissipated in the cavity wall (or in the coupler load):

$$
I V_{1}=V_{1}^{2} / R_{1}=\omega U / Q,
$$

where $Q$ is a quality factor and $\omega$ is the angular resonance frequency of the mode. Finally, using Eq. (5), we get

$$
R_{1} / Q=V_{1}^{2} / \omega U
$$

The stored field energy $(U)$ in the mode is proportional to the square of the field amplitude $U \propto B^{2}$. Therefore, $R_{1} / Q$ is independent of the field amplitude (because $V_{1} \propto B$ ).

The Panofsky-Wenzel theorem describes the ratio of transverse and longitudinal fields as

$$
R_{\perp} / R_{1}=P_{\perp} c / V_{1}=c / \omega y .
$$

Usually, the coupling impedances of the cavity modes are calculated in a "thin cavity approximation," the integration path to calculate the energy gain of the bunch is a straight line with a fixed offset to the cavity axis (see Fig. 1). It is usually assumed that the bunch travels at the speed of light $c$. At the cavity exit, the bunch receives both longitudinal and transversal kicks characterized by a change of energy $\Delta E$ and transversal momentum $(\Delta P c)$. Both parameters depend on the $\operatorname{rf}$ phase $\varphi$ of the mode:

$$
\begin{aligned}
\Delta E & =V_{1} \cdot \cos (\varphi) \\
\Delta P c & =P_{\perp} c \cdot \sin (\varphi) .
\end{aligned}
$$

Here, $V_{1}$ is the "longitudinal" energy in $\mathrm{eV}$, and $P_{\perp} c=V_{\perp}$ is called the "transverse energy."

In this paper, the impedances are expressed in Ohms. In practice, offset-independent impedances of the dipole modes are often used, i.e., $R_{I I} / 2 y^{2}\left[\mathrm{Ohm} / \mathrm{m}^{2}\right]$ and $R_{\perp} / 2 y[\mathrm{Ohm} / \mathrm{m}]$.

\section{B. The source of BBU instability in cavities}

We will analyze the interaction of a beam with any dipole field excited by an external source. While electron bunches are launched at a fixed main mode phase, the phase of dipole modes changes from shot to shot as $\Delta \varphi=$ $n \omega T=2 \pi n \frac{f}{f_{0}}$, i.e., the dipole modes all have launch phases $(0-2 \pi)$ with equal probability if there is no resonance between the main mode $\left(f_{0}\right)$ and the $\operatorname{HOM}(f)$. Here, $T$ is the repetition time, and $n$ is an integer. Therefore, the energy transfer to the HOM in Eq. (8) averages to zero. In any rf gun cavity with an initial beam offset $(y)$ shown in Fig. 2 by the blue curve, a net energy loss of particles

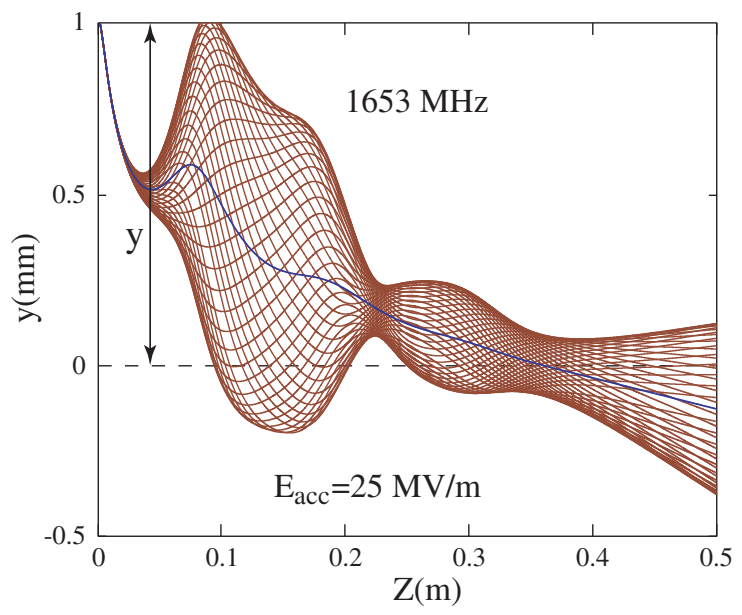

FIG. 2. The actual trajectories in the rf gun cavity dependent on the beam launch phase relative to the $1653-\mathrm{MHz}$ dipole mode $(B=0.001 \mathrm{~T})$. The fundamental mode at $1300 \mathrm{MHz}$ has an accelerating field of $25 \mathrm{MV} / \mathrm{m}$. The blue trajectory depicts the trajectory without the dipole mode $(B=0 \mathrm{~T})$. 
emitted over all phases is possible if the change in trajectories under the influence of the dipole fields is taken into account. These oscillating trajectories are depicted by red curves in Fig. 2, i.e., there exists a phase independent component in Eq. (8) that cannot be averaged to zero. Because of energy conservation, the energy of the dipole field changes by the same amount of energy as the particle lost, which is then compensated by the external source. If the field is excited by the beam when the external source is absent, this phase independent component can drive BBU instability.

Intuitively, BBU instability can be explained as follows: the average particle energy exchanged due to the HOM is proportional to the trajectory oscillation $\delta y$ and the dipole field strength $(\delta y \cdot B)$. The trajectory oscillation, if the HOM field is taken into account, is also proportional to the dipole field strength $(\delta y \propto B)$. Therefore, there will be components in Eq. (8) proportional to $B^{2}$, i.e., to the stored energy in the dipole mode $U$. Below, these components of the energy kick are denoted by $V_{0}$ and $V_{2}$. The threshold current occurs when the power fed into the mode $\left(I V_{0}+I V_{2}\right)$ equals the mode power dissipation (the dissipation is also proportional to $B^{2}$ ). This situation represents the fundamental condition for the instability of a bunched or DC beam current interacting with dipole or monopole HOMs, as described in [6]. The dipole mode interaction is unique due to the presence of the $V_{2}$ component in Eq. (8).

Because of the oscillation of the trajectory, as is analytically proven in Ref. [4], the energy dependence of Eq. (8) is modified by two additional Fourier-like components. (Other Fourier components appear if the nonlinearity of dipole fields near the axis are taken into account.)

$$
\Delta E=V_{0}+V_{1} \cos (\varphi)+V_{2} \cos (2 \varphi+\Phi) .
$$

Here, $\varphi$ is the dipole mode phase related to the moment when the excited voltage $V_{1}$ has a maximum, and $\Phi$ is a constant phase shift. The components $V_{0}$ and $V_{2}$ are proportional to $B^{2}$ of the dipole HOM and are offset independent. The field integration is made along the curved trajectory taking into account the changed particle velocity driven by the accelerating field of the fundamental mode without the dipole HOM. This trajectory is shown in Fig. 2 by the blue curve.

We consider the appearance of the additional components $\left(V_{0}, V_{2}, \Phi\right)$ in Eq. (10) as the influence of dynamic coupling between the beam and the HOM due to trajectory oscillations. This dynamic coupling impedance can cause BBU instability in a cavity.

Because Eq. (9) is obtained by integrating the $E_{y}$ and $B_{x}$ components of the dipole fields, which are independent of the trajectory offset, the form of Eq. (9) is changed only by the addition of a constant phase shift in the argument of the sine that appeared due to the variable particle velocity. The magnitude of $P_{\perp} c$ in Eq. (9) will be some factor different, and the transverse impedance must be multiplied by this factor according to Eq. (4).

The dynamic aspects of Eq. (10) [4] of transverse particle motion along a near-straight line in a vertically polarized field driven by rf fields are considered:

$$
\frac{d}{d t} m \dot{y} \gamma=e E_{y} \cos (\omega t+\varphi)+e \beta c B_{x} \sin (\omega t+\varphi) .
$$

The general solution of Eq. (11) for the particle trajectory in the dipole field is

$$
\begin{aligned}
y(z, t)= & \frac{e}{m \gamma \omega^{2}} c B\left[Y_{0}(z) \cos (\omega t+\varphi)\right. \\
& \left.+Y_{2}(z) \sin (\omega t+\varphi)\right]+y_{1},
\end{aligned}
$$

where $B$ is the on-axis maximal $B_{x}$ dipole field, $y_{1}$ is the initial $y$ offset, and the complex dimensionless function $\dot{Y}=Y_{0}(z)+j Y_{2}(z)$ is a solution of a complex differential equation:

$$
\frac{\partial^{2} \dot{Y}}{\partial \psi^{2}}-2 j \frac{\partial \dot{Y}}{\partial \psi}-\dot{Y}=E_{y}(\psi) / c B+j \beta B_{x}(\psi) / B,
$$

where $\psi=k z, k=\omega / \beta c$, and $\beta c=v$ is the particle velocity.

Integrating the electric dipole field $E_{z}$ and $E_{y}$ along this trajectory, we obtain the components of Eq. (10):

$\Delta E=\frac{e c^{2} B^{2}}{2 m \gamma \omega^{2}}\left[I_{0}-I_{2} \cos (2 \varphi+\Phi)\right]+c B y_{1} I_{1} \cos (\varphi)$.

It is proven by numerical calculations that, in the case of $\mathrm{rf}$ gun dynamics in which $\beta c$ changes due to a strong acceleration from the nonrelativistic to the relativistic regime and the trajectory is not a straight line, this constant phase $\Phi$ is offset independent. Furthermore, it depends only on the dipole and accelerating field configuration, and each mode has a certain value from 0 to $2 \pi$. The dimensionless cumulative constants $I_{0}, I_{1}$, and $I_{2}$ also depend only on dipole and accelerating field configuration and are offset independent. For example, $I_{0}$ is given as follows:

$$
\begin{aligned}
I_{0}= & \int_{0}^{k L} \frac{\partial\left(E_{z} / c B\right)}{\partial k y} Y_{0}(\psi) d \psi+\int_{0}^{k L}\left(E_{y} / c B\right) \frac{\partial Y_{0}(\psi)}{\partial \psi} d \psi \\
& +\int_{0}^{k L}\left(E_{y} / c B\right) Y_{2}(\psi) d \psi
\end{aligned}
$$

where $L$ is the cavity length.

The condition of the excited equal-to-dissipated power gives the threshold current [4],

$$
I_{\mathrm{thr}} Q=-\frac{\omega U}{V_{0}}=\frac{-2 m \gamma}{e I_{0}} \omega^{3}\left(\frac{U}{c^{2} B^{2}}\right) .
$$

The energy change (see Fig. 3, Table I) is numerically calculated according to the algorithm described in Ref. [4]. The calculated values fit the analytical dependence of Eq. (10) within an accuracy of approximately $1 \%$. We can assume without a loss of generality that the focusing 


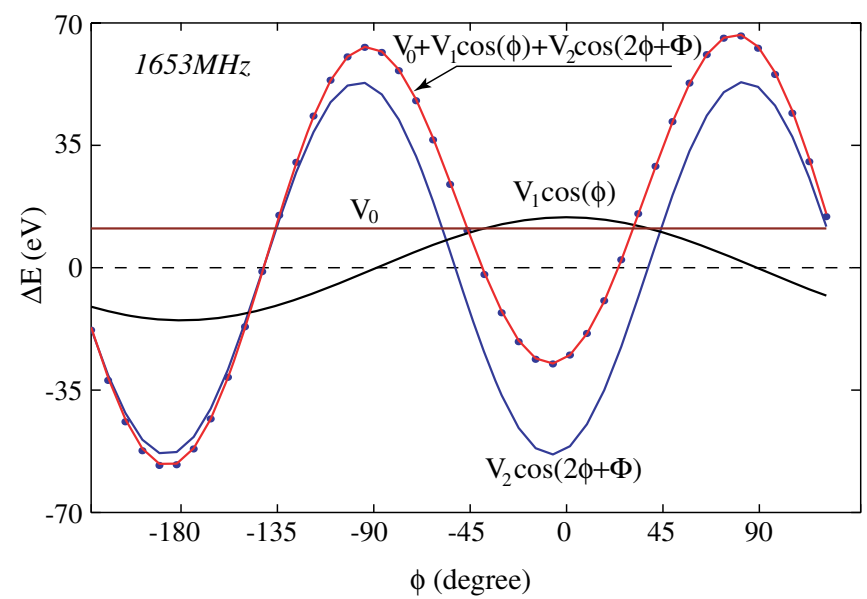

FIG. 3. The numerically calculated energy kick and voltage components of Eq. (10) for the dipole 1653-MHz mode depicted by a dotted line and fitted by harmonic functions.

TE mode fields do not change the form of Eq. (10), even if the averaging of $\Delta E$ over all phases of the TE mode is applied. This is proved analytically and numerically in [4].

Hypothetically, approximately half of all dipole HOMs can be unstable, i.e., can experience exponential growth. This fact is obvious in a thin-cavity model of a recirculating linac in which the beam and the cavity form a feedback loop that closes upon the return of the beam to the same cavity on a subsequent pass [7]. The physics of all of these effects is fundamentally the same, and the threshold current occurs when the power fed into the mode equals the mode power dissipation. Without a loss of generality, we can assume the recirculating linac model is a two-cell cavity with a long distance between cells in which the longitudinal coupling impedance depends on the squared beam trajectory offset. Threshold currents in Ref. [7] are similar to Eq. (16) (first term) if we replace $(R / Q)_{d}$ with our impedances defined in Ohms. The threshold current becomes the following:

$$
I_{\mathrm{th}} Q=\frac{-2 \omega p}{q c\left(R_{I I} / Q\right) m^{*} \sin \left(\omega T_{r}\right)} .
$$

Here, $T_{r}$ in a multicell cavity is the time it takes a bunch to travel between the centers of the two cells. The phase delays $\omega T_{r}$ for each HOM are unique, and statistically, half of them will have a negative sign indicating the instability.

\section{Derivation of coupling impedance formulas in a beam-loaded cavity}

The coupling impedances of Eqs. (6) and (7) obtained by integration over a real trajectory depends on the beam current because the excited dipole HOM field changes these trajectories. Although the term "impedance" is considered usually to be current independent, we will refer to it as "impedance" because we assume here the average beam current is constant in time.

This current-dependent longitudinal impedance and induced voltage are denoted here by $R_{I I}$ and $V_{I I}$, respectively. Thus, Eq. (6) must be rewritten as

$$
R_{I I} / Q=V_{I I}^{2} / \omega U
$$

At low beam current, we have $V_{0} \approx V_{2} \approx 0$ and $V_{I I} \approx V_{1}$. Therefore, the coupling impedance at low beam current is close to a constant component $\left(R_{1}\right)$, independent of the beam current and defined by Eq. (6).

By definition, the excited voltage has the opposite sign than the particle energy loss, i.e., $V_{q}=-\Delta E$. Furthermore, the phase $\varphi$ of the excited mode must be set relative to the excited voltage, i.e., in Eq. (10), $\varphi \rightarrow \varphi+\pi$. Assuming the above and Eq. (10), we derive the excited voltage as

$$
V_{q}=V_{1} \cos (\varphi)-V_{0}-V_{2} \cos (2 \varphi+\Phi) .
$$

Here, we assume the analytic model of HOM excitation by pointlike bunches periodically moving (with period $T$ ) through the cavity. After a bunch with a charge $q$ leaves

TABLE I. The numerically calculated voltage components of Eq. (10) for $E_{\text {acc }}=25 \mathrm{MV} / \mathrm{m}$. $B$ is the maximal on-axis dipole field. $N$ is the serial number of the calculated dipole mode.

\begin{tabular}{cccccc}
\hline \hline$N$ & $F(\mathrm{MHz})$ & $V_{0} / B^{2}\left(\mathrm{~V} / \mathrm{mT}^{2}\right)$ & $V_{1} / y / B(\mathrm{~V} / \mathrm{mm} / \mathrm{mT})$ & $V_{2} / B^{2}\left(\mathrm{~V} / \mathrm{mT}^{2}\right)$ & $\Phi$ degrees \\
\hline 1 & 1653 & 11.3 & 14.7 & 53.2 & -166 \\
2 & 1724 & -400 & 447 & 589 & 14.3 \\
3 & 1766 & 1118 & 616 & 920 & 127 \\
4 & 1814 & 90.2 & 104 & 73.3 & -148 \\
5 & 1864 & 12.6 & 136 & 105 & 110 \\
6 & 1873 & 4.86 & 29.5 & 58.7 & -127 \\
7 & 1887 & -22.2 & 82.5 & 24.3 & 63 \\
12 & 2699 & 14.5 & 53.2 & 24.6 & 38.7 \\
20 & 3159 & 10.3 & 68.5 & 20.6 & 112 \\
37 & 4466 & -5.0 & 195 & 27.5 & 118 \\
89 & 6709 & 4.43 & 167 & 2.41 & 109 \\
\hline \hline
\end{tabular}


the cavity, a beam-induced voltage with a resonance HOM frequency $(\omega)$

$$
\Delta V_{q}=q\left(R_{I I} / Q\right) \omega e^{i \omega t-\omega t / 2 Q}=I\left(R_{I I} / Q\right) \omega T e^{i \omega t-\omega t / 2 Q}
$$

remains in each mode. By superposition, $\Delta V_{q}$ in a cavity is the same whether a generator or previously self-excited voltage is present. This is true even if we have a situation with nonrigid bunches, as the $R_{I I} / Q$ value depends on the bunch trajectory in the cavity that, due to large currents, depends on the squared excited field amplitude and on its phase $\varphi$. The total excited voltage, $\dot{V}$, is the sum of an infinite number of bunch-induced voltages representing a geometric progression:

$$
\dot{V}=\Delta V_{q} e^{i \omega t-\omega t / 2 Q} \sum_{n=0}^{\infty}\left(e^{i \omega T-\omega T / 2 Q}\right)^{n}=\frac{\Delta V_{q} e^{i \omega t-\omega t / 2 Q}}{1-e^{i \omega T-\omega T / 2 Q}},
$$

where $n$ is the serial number of the previously passed bunch through the cavity at the time, $n T+t$, back from the recently $(n=0)$ passed bunch moment $t(0<t<T)$. This excitation process is depicted in the complex plane, as shown in Fig. 4, at a moment $t=0$, just after the recent bunch leaves the cavity. The previously excited voltage $(n=1)$ in the cavity at that moment is $\dot{V}_{-1}=\dot{V}-\Delta V_{q}$.

According to the fundamental theorem of beam loading, the charge itself sees the $1 / 2$ fraction of $\Delta V_{q}$, i.e.,

$$
\dot{V}_{q}=\dot{V}-\frac{1}{2} \Delta V_{q}=\frac{1}{2} I\left(R_{I I} / Q\right) \omega T \frac{1+e^{i \omega T-\omega T / 2 Q}}{1-e^{i \omega T-\omega T / 2 Q}},
$$

where $\Delta V_{q}$ is replaced by Eq. (20). The hodograph of $\dot{V}_{q}$ at $I\left(R_{I I} / Q\right)=$ const obtained by varying $\omega T$ is depicted in

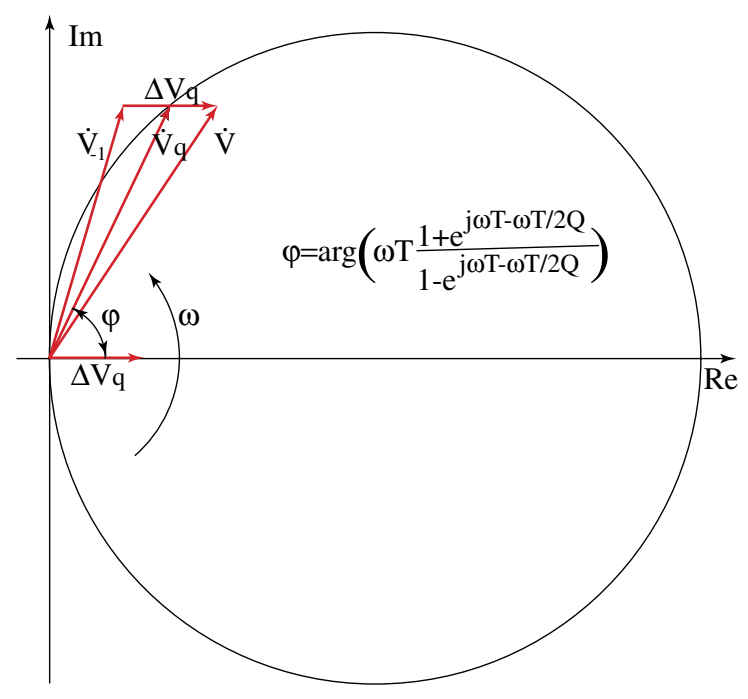

FIG. 4. The complex plane with voltages excited by a series of pointlike bunches entering the cavity at regular intervals.
Fig. 4. It is a cycle with $\operatorname{Re}\left(\dot{V}_{q}\right)=I R_{I I} \cos (\varphi)^{2}$ that is fully equivalent to the hodograph of a resistor, inductance, and capacitor (RLC) oscillating circuit, where $\varphi$ is the argument of $\dot{V}_{q}$ that corresponds to the angle between the beam current and the excited voltage vectors. The real part of $\dot{V}_{q}$ is identical to $V_{q}$ of Eq. (19).

This implies that the excited HOM field amplitude in the cavity is proportional to $V_{1} \cos (\varphi)$. Therefore, the first term in Eq. (19) is $\left[V_{1} \cos (\varphi)\right] \cos (\varphi)=V_{1} \cos (\varphi)^{2}$ and $V_{0}+V_{2} \cos (2 \varphi+\Phi)=\alpha_{0}\left[V_{1} \cos (\varphi)\right]^{2}+\alpha_{2}\left[V_{2} \cos (\varphi)\right]^{2} \times$ $\cos (2 \varphi+\Phi)$ because the other two terms of Eq. (19) are proportional to the squared HOM field amplitude. The factors $\alpha_{0}$ and $\alpha_{2}$ are constants. After inserting the above to the equation $\operatorname{Re}\left(\dot{V}_{q}\right)=V_{q}$ and using Eq. (19), we obtain

$$
\begin{aligned}
\operatorname{Re}\left(\dot{V}_{q}\right)= & I R_{I I} \cos (\varphi)^{2} \\
= & V_{1} \cos (\varphi)^{2}-\alpha_{0}\left[V_{1} \cos (\varphi)\right]^{2} \\
& -\alpha_{2}\left[V_{1} \cos (\varphi)\right]^{2} \cos (2 \varphi+\Phi)
\end{aligned}
$$

From Eq. (23), after dividing it by $\cos (\varphi)^{2}$, we get the following:

$$
I R_{I I}=V_{I I}=V_{1}-V_{0}-V_{2} \cos (2 \varphi+\Phi) .
$$

The equivalence of such a current-dependent beamcavity interaction with electric processes in an RLC circuit has an average current-dependent $R_{I I} / Q$ that is uniquely determined by Eq. (24). Therefore, we can consider the application of Eq. (18) to be feasible.

Without a loss of generality, it can be assumed in practice that $V_{2} \cos (2 \varphi+\Phi) \approx-V_{2} \cos (\Phi)$ because as a rule, there are no resonances with the HOM, i.e., $\varphi \approx \pm \pi / 2$. In reality, the probability of the resonance case is on the order $1 / Q$ in magnitude. We can consider in this case that the current-dependent excitation process does not differ from the usual process but has the possibility to be unstable.

To obtain the coupling impedance at a higher beam current, we can formulate using Eqs. (18), (6), and (24):

$$
\begin{aligned}
R_{I I} / Q & =\left[V_{1}-V_{0}-V_{2} \cos (2 \varphi+\Phi)\right]^{2} / \omega U \\
& =\left(R_{1} / Q\right)\left(1-\frac{V_{0}+V_{2} \cos (2 \varphi+\Phi)}{V_{1}}\right)^{2} .
\end{aligned}
$$

Because the dissipated power $(\omega U / Q)$ must equal the excitation power $\left(I V_{I I}\right)$ implied by Eq. (24), it follows that

$$
\begin{aligned}
\omega U / Q & =\left[V_{1}-V_{0}-V_{2} \cos (2 \varphi+\Phi)\right] I \\
& =(\omega U / Q)\left(I / I_{\mathrm{th}}\right)\left(1-\frac{V_{1}}{V_{0}+V_{2} \cos (2 \varphi+\Phi)}\right) .
\end{aligned}
$$

Here, we introduce a more accurate threshold current definition (depending on the period $T$ through the phase $\varphi$ ) that differs from the definition obtained in Ref. [4] [first term of Eq. (16)]:

$$
I_{\text {th }} Q=-\frac{\omega U}{V_{0}+V_{2} \cos (2 \varphi+\Phi)} .
$$


The combination of these last three equations gives

$$
R_{I I} / Q=\frac{R_{1} / Q}{\left(1-I / I_{\mathrm{th}}\right)^{2}} .
$$

Recall that the change in the transverse momentum $\left(P_{\perp} c\right)$ obtained by integrating the fields $E_{y}$ and $B_{x}$ along trajectories does not depend on the trajectory oscillation $\delta y$ because these fields are offset independent within these approximations. Therefore, the linear dependence of $P_{\perp} c$ on the field amplitude or on the square root of the stored $\mathrm{rf}$ energy $U^{1 / 2}$ is conserved. By Eq. (4), the linear dependence is then

$$
\frac{P_{\perp} c}{\sqrt{\omega U}}=\frac{R_{\perp} / Q}{\sqrt{R_{I I} / Q}}=\frac{\left(R_{\perp} / Q\right)_{\mid I \approx 0}}{\sqrt{R_{1} / Q}},
$$

where $\left(R_{\perp} / Q\right)_{\mid I \approx 0}$ is the transverse impedance at a low beam current, which can be numerically calculated. Using Eqs. (6) and (7),

$$
\left(R_{\perp} / Q\right)_{\mid I \approx 0}=\frac{P_{\perp} c V_{1}}{\omega U} .
$$

Finally, the equation of the transverse coupling impedance using Eqs. (28) and (29) is

$$
R_{\perp} / Q=\frac{\left(R_{\perp} / Q\right)_{\mid I \approx 0}}{\left|1-I / I_{\mathrm{th}}\right|} .
$$

Clearly, because stable dipole modes must lose stored energy to the beam during the beam-dipole mode interaction, then $V_{0}+V_{2} \cos (2 \varphi+\Phi)>0$, i.e., the threshold current in Eq. (27) is negative, and there are plus signs in Eqs. (28) and (31). It follows that coupling impedances of stable dipole modes will decrease for large beam currents. The opposite is true for unstable mode impedances, which become infinite at the threshold current. We have to note that the current dependency of Eq. (31) is experimentally confirmed in Ref. [7].

Table II lists the calculated transverse impedances for the Fourier components of a low beam current $\left(R_{\perp} / Q / 2 y\right)$

TABLE II. Transverse impedances $R_{\perp} / Q[\Omega / \mathrm{m}]$ for $E_{\text {acc }}=$ $25 \mathrm{MV} / \mathrm{m}$ and threshold currents $[\mathrm{A}]$ of Eq. (30).

\begin{tabular}{lcccc}
\hline \hline$N$ & $F[\mathrm{MHz}]$ & CLANS $[\Omega / \mathrm{m}]$ & Eq. $(30)[\Omega / \mathrm{m}]$ & $I_{\text {th }} Q[\mathrm{~A}]$ \\
\hline 1 & 1653 & 84 & 4.2 & $-2.8 \times 10^{5}$ \\
2 & 1723 & 241 & 255 & $+1.9 \times 10^{4}$ \\
3 & 1765 & 1940 & 719 & $-1.1 \times 10^{4}$ \\
4 & 1814 & 371 & 74.5 & $-4.5 \times 10^{4}$ \\
5 & 1864 & 289 & 143 & $-1.4 \times 10^{5}$ \\
6 & 1873 & 100 & 14.2 & $-1.1 \times 10^{5}$ \\
7 & 1887 & 57 & 49.3 & $+2.5 \times 10^{5}$ \\
12 & 2698 & 208 & 19.6 & $+1.3 \times 10^{6}$ \\
20 & 3158 & 51 & 18.1 & $-6.4 \times 10^{5}$ \\
37 & 4466 & 12 & 34.9 & $-1.5 \times 10^{6}$ \\
89 & 6707 & 75 & 41.0 & $-5.9 \times 10^{5}$ \\
\hline \hline
\end{tabular}

of Eq. (30) with its threshold currents multiplied by the quality factor. No resonance is assumed, i.e., $\varphi \approx \pm \pi / 2$. The impedances, as calculated using CLANS2 according to the accepted theory (a straight-line trajectory approximation of particles at the velocity of light), are also presented for comparison. Interestingly, these impedances, as a rule, are significantly larger than those calculated for the rf gun.

Because quality factors directly affect the threshold currents, there is a necessity to decrease the quality factors as much as possible to obtain stability at high current beams. Below, we will analyze these possibilities.

\section{TRAPPED DIPOLE MODE FEATURES}

Trapped modes are an important class of modes whose frequencies lie above the cutoff frequency of the beam pipe but whose damping by a beam-tube load is very weak because they poorly couple to the tube. Hence, an analysis of their features is important for the determination of BBU instabilities.

We have calculated the dipole HOM field distributions up to $8 \mathrm{GHz}$ for a FZD-like, $3 \frac{1}{2}$ cell, rf gun cavity using the 2D CLANS2 field solver [8] and consider eleven HOMs with the greatest $Q / \omega^{3}$ values according to Eq. (16).

An external load (see $Q_{\text {ext }}$ in Fig. 5) at the end of the beam pipe is responsible for the dipole mode damping. Given properly chosen characteristics of the load in the calculation, the incident $\mathrm{rf}$ power $(P)$ is dissipated in the load without reflection. Because trapped modes poorly couple to the pipe, this power is small, and these modes will have higher qualities.

To simplify the study of the HOM properties, the transverse coupling impedances $\left(R_{\perp}\right)$ in this section were calculated using the CLANS2 code using the conventional formalism for ultrarelativistic particles [9]. The properties of the modes were studied as a function of changing boundary conditions. Here, we assume that the actual impedances for $\mathrm{rf}$ gun beams considered in the following sections react to system changes (e.g., cavity length) in a similar manner.

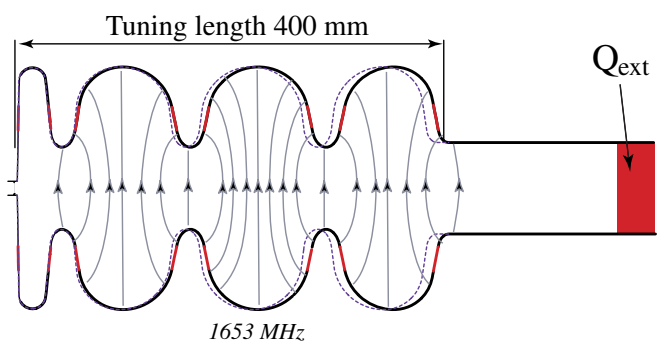

FIG. 5. The $3 \frac{1}{2}$-cell cavity of an FZD-like SRF gun with a perfect rf load $Q_{\text {ext }}$. The electric field lines of the $1653-\mathrm{MHz}$, vertically polarized dipole mode are shown. 


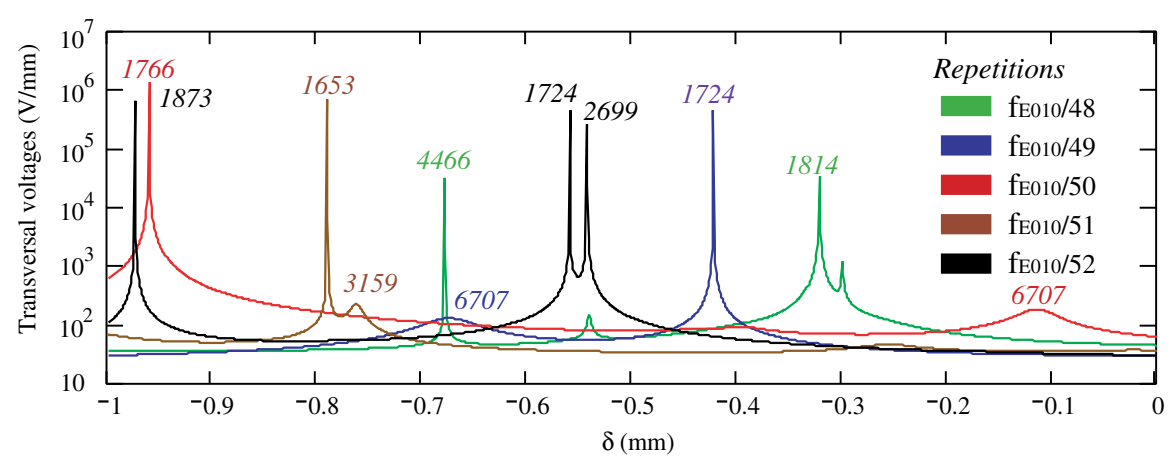

FIG. 6. Transverse voltages of all dipole HOMs excited by a 2-mA beam at different bunch repetition frequencies versus the cavity deformation ( $\delta$ parameter). The numbers over the peaks are the frequencies of excited dipole HOMs.

\section{A. Dipole mode amplitudes versus cavity detuning}

In this section, we examine the dipole HOM amplitude changes that follow from a cavity-length detuning. Typically, the length of the cavity is mechanically modified to match the fundamental frequency to the master clock. In the simulations, we model the cavity shape changes by changing the inclination of the geometry marked in red in Fig. 5. Other sections remain unchanged. Clearly, all other HOM frequencies will also be changed. Because the bunch repetition period is coupled to the fundamental frequency by $T=n / f_{0}$ ( $n$ is an integer), it is also detuned.

The sum of all of the dipole HOM voltages, each calculated by Eq. (22) with different repetitions $n$, is depicted in Fig. 6. We see in Fig. 6 that some HOM voltages increase significantly at some cavity deformations. This occurs when the resonances approach the beam harmonics $\left(\omega_{i} T / 2 \pi\right.$ is integer). This result shows that HOMs can affect the beam quality for some cavity shapes (cavity length) and repetition frequencies. These effects can be avoided by switching between different repetitions rates, as demonstrated in Fig. 6 in which the excited spectra for different repetition rates are depicted.

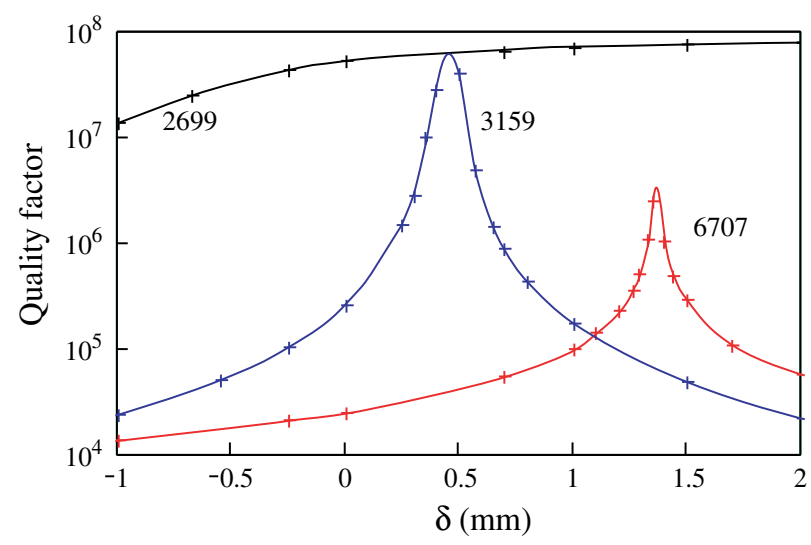

FIG. 7. Trapped dipole HOM external quality factors versus the cavity longitudinal deformation.

\section{B. Trapped HOM quality factors versus detuning}

The loaded quality factors of some trapped HOMs increase significantly during detuning. The external quality factors of three trapped dipole HOMs versus cavity deformation is depicted in Fig. 7. This indicates the high coupling sensitivity of some trapped HOMs to the beam pipe on the cavity deformation. As shown in Fig. 7, some trapped HOMs can increase their $Q$ factor significantly and can potentially drive BBU instability. It should be noted that frequency detuning of all HOMs $f_{i}(\delta)$ remains close to linear.

\section{DYNAMICS SIMULATION OF FZD-LIKE RF GUN CAVITY}

In this section, we analyze the impact of the additional focusing of the bunch by an externally excited TE mode on BBU instability in the FZD-like superconducting rf (SRF) gun. Initially, it was suggested in Ref. [10] to use the field of an externally excited TE mode for emittance compensation instead of a solenoid. In this way, the (relatively high) magnetic field of the solenoid near the superconducting gun cavity is avoided, and booster cavities can be placed closer to the gun. However, the focusing by this mode modifies the BBU threshold current of other modes.

Note that the initial phasing between the TE and accelerating mode is time dependent but this does not influence the beam quality because the TE mode frequency (3781 MHz) is large (see Ref. [11]). Because the dipole HOM frequencies are large, the BBU instability is affected, and hence, the voltages calculated with Eq. (10) were averaged over all TE mode phases.

In Table III, the calculated threshold currents multiplied by the quality factors are presented for selected dipole HOMs. Three of them are unstable (positive thresholds). Focusing by the TE 3781-MHz mode suppresses instability, but there are dipole modes $(1653,1864$, and 3159 in Table III) that react little to focusing or even become unstable due to this focusing. Presumably, this effect is due to insufficient focusing, as this TE mode peaks in the first accelerating cell and then decreases for subsequent cells in which the dipole modes progressively increase 
TABLE III. Threshold currents multiplied by the quality factors $I_{\text {th }} \cdot Q[A]$ of dipole modes at different focusing strengths $B_{\mathrm{TE}}[\mathrm{T}]$ for the $3781-\mathrm{MHz}$ mode [see Eq. (27)]. The negative ones correspond to stable modes. $E_{\text {acc }}=25 \mathrm{MV} / \mathrm{m}$.

\begin{tabular}{lrrrrr}
\hline \hline$N$ & $F \mathrm{MHz}$ & \multicolumn{1}{c}{$B_{\mathrm{TE}}=0 \mathrm{~T}$} & $B_{\mathrm{TE}}=0.1 \mathrm{~T}$ & $B_{\mathrm{TE}}=0.2 \mathrm{~T}$ & \multicolumn{1}{c}{$B_{\mathrm{TE}}=0.3 \mathrm{~T}$} \\
\hline 1 & 1653 & $-2.8 \times 10^{5}$ & $3.6 \times 10^{5}$ & $3.2 \times 10^{5}$ & $1.8 \times 10^{5}$ \\
2 & 1724 & $1.9 \times 10^{4}$ & $3.0 \times 10^{4}$ & $8.4 \times 10^{5}$ & $-8.2 \times 10^{4}$ \\
3 & 1766 & $-1.1 \times 10^{4}$ & $-1.1 \times 10^{4}$ & $-2.1 \times 10^{4}$ & $-1.1 \times 10^{5}$ \\
4 & 1813 & $-4.5 \times 10^{5}$ & $-7.3 \times 10^{4}$ & $-1.1 \times 10^{5}$ & $-1.1 \times 10^{5}$ \\
5 & 1864 & $-1.4 \times 10^{5}$ & $-7.2 \times 10^{4}$ & $2.7 \times 10^{5}$ & $6.2 \times 10^{5}$ \\
6 & 1873 & $-1.1 \times 10^{5}$ & $-1.1 \times 10^{5}$ & $-6.2 \times 10^{4}$ & $-1.1 \times 10^{5}$ \\
7 & 1887 & $2.5 \times 10^{5}$ & $2.0 \times 10^{5}$ & $-5.6 \times 10^{6}$ & $2.6 \times 10^{7}$ \\
12 & 2699 & $1.3 \times 10^{6}$ & $-1.0 \times 10^{7}$ & $-4.3 \times 10^{5}$ & $-2.8 \times 10^{5}$ \\
20 & 3159 & $-6.4 \times 10^{5}$ & $-7.0 \times 10^{5}$ & $2.5 \times 10^{6}$ & $6.7 \times 10^{6}$ \\
37 & 4466 & $-1.5 \times 10^{6}$ & $-1.1 \times 10^{6}$ & $-6.0 \times 10^{5}$ & $-3.6 \times 10^{5}$ \\
89 & 6709 & $-5.9 \times 10^{5}$ & $-5.9 \times 10^{5}$ & $-5.9 \times 10^{5}$ & $-6.1 \times 10^{5}$ \\
\hline \hline
\end{tabular}

(see Fig. 8 and Ref. [10]). We recall that the focusing effect distribution along the axis depends on the field amplitude distribution $B_{\mathrm{TE}}(z)^{2}$ [11]. In such a scenario, the trajectories depicted in Fig. 2 are condensed in the first full cell but fan out in subsequent cells, i.e., there is a defocusing effect in the last cells.

To avoid this problem, a specially shaped rf gun cavity design can be applied. In this cavity, each of the three full cells has equal amplitudes for the fundamental as well as for a focusing TE mode. The sketch of such a cavity with a $\mathrm{TE}_{021}$ mode of $\pi$ type is depicted in Fig. 9. The accelerating mode remains at $1300 \mathrm{MHz}$ and has equal amplitudes in each cell. The beam is focused in each cavity cell, and therefore, BBU instability can be effectively suppressed. The impact on the threshold currents must be calculated in a new simulation because the dipole HOM spectrum for

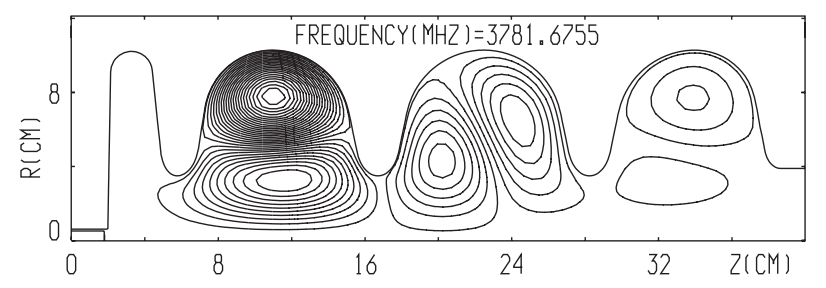

FIG. 8. Magnetic field lines of the $\mathrm{TE}_{021}$ focusing mode in the rf gun

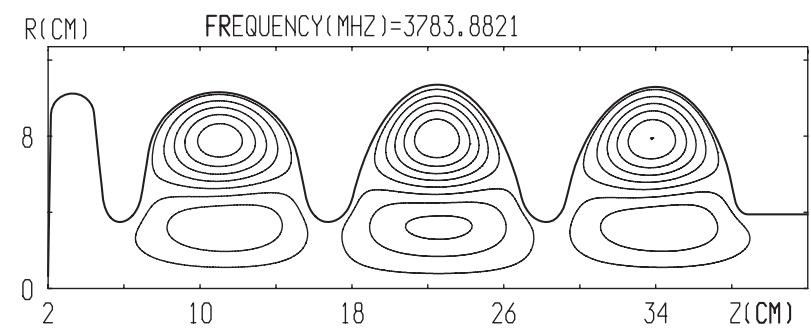

FIG. 9. The $\mathrm{TE}_{021}$ focusing $\pi$ mode in all three cells. such a cavity differs significantly. The impact will be discussed in another paper.

\section{NEW DESIGNS FOR HOM DAMPING}

In this section, we propose two novel design schemes to damp the higher modes.

Figures 10 and 11 depict two schemes for HOM damping. The newly proposed concept of HOM damping is consistent with the higher-order TE focusing mode. The HOM damping effectively suppresses both monopole and dipole HOMs and couples to some trapped HOMs. A special insert presents a coaxial line to the cavity with a matched ferrite load at the opposite end, thereby ensuring that both monopole and dipole modes propagate down the line for heavy damping in the load. A similar design was

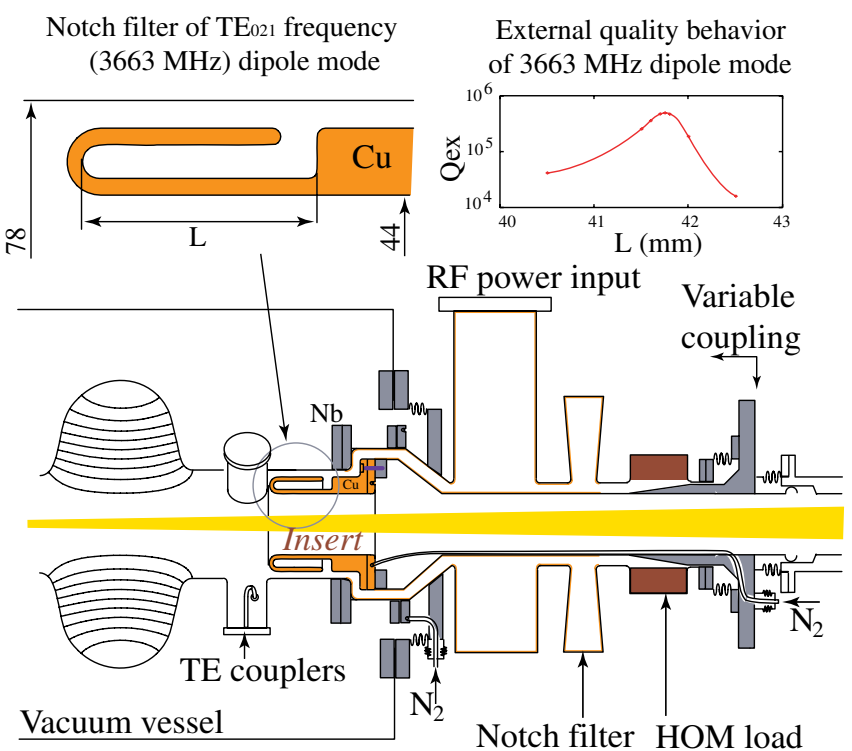

FIG. 10. HOM-damping scheme with the rf power input and the notch outside the cryostat. 


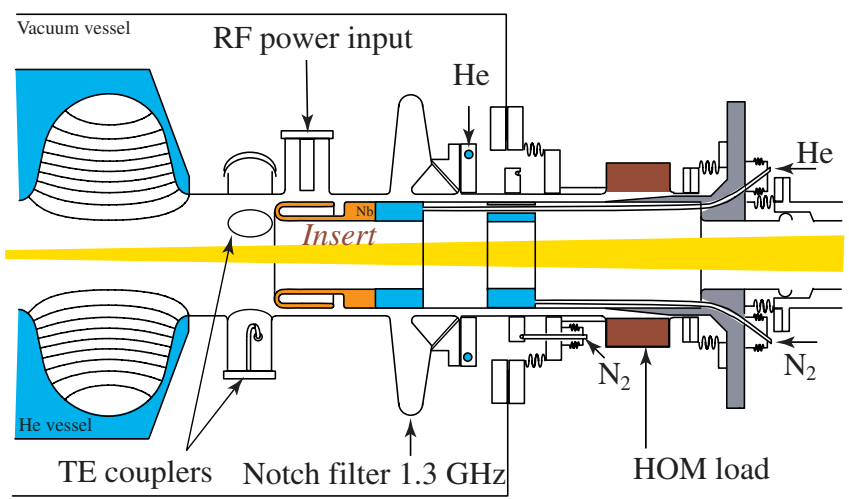

FIG. 11. HOM-damping scheme with a superconducting insert, a conventional rf power input, and superconducting notches inside the cryostat.

employed to successfully damp HOMs in the normal conducting VEPP2000 cavity [12]. The insert also serves as the fundamental rf power input coupler mode, providing a highly symmetric system. It could be longitudinally tuned by a motor to change the external quality factor to match the cavity to the rf source. The insert is thermally isolated such that it can be cooled with liquid nitrogen or, as shown in the second variant in Fig. 11, with helium.

There are two notch filters. One of them isolates the fundamental rf power mode from the HOM load while the second isolates the TE focusing mode (see Figs. 10-12). The field of the TE mode is axiasymmetric due to the TE couplers, and a dipolelike component of the field appears [13]. The notch filter changes the field distribution of the dipolelike component to prevent the coupling of the TE mode to the HOM load. The distribution of the dipole electric field in the notch filter is shown in Fig. 12. The rf field is zero at the entrance of the following coax line.

Besides the power losses of the TE mode in the superconducting wall, the external quality factor of the TE mode is limited (see the insert in Fig. 10) by the dissipated power of the dipolelike component in the normal conducting notch. This limited quality is much higher in the superconductive notch variant. The rf power of the TE focusing mode needed to operate the cavity with $B_{\mathrm{TE}}=0.1 \mathrm{~T}$ can

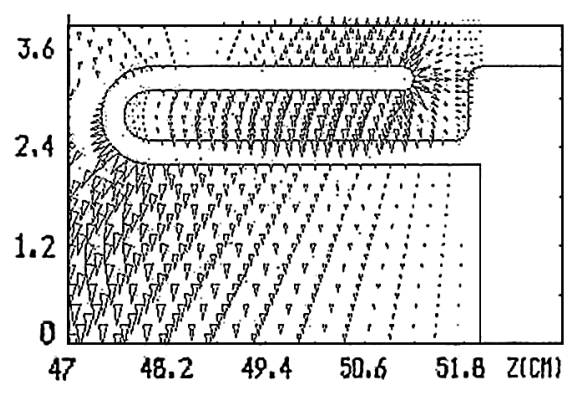

FIG. 12. The electric field distribution in the notch filter (CLANS2 output). be found for this rf gun from the numerically calculated formula,

$$
P[W]=2.7+2.6 \times 10^{5} K_{\mathrm{tr}},
$$

where the first term is the dissipated TE mode power in the cavity wall. The second term is the dipole mode power dissipated in the copper notch filter, which remains at the temperature of liquid nitrogen. $K_{\mathrm{tr}}=\left(B_{\mathrm{dip}} / B_{\mathrm{TE}}\right)^{2}$ is the coefficient of TE focusing-to-dipole mode transformation due to the asymmetry, and $B_{\text {dip }}$ is an axis field of the dipole field. We assume here for a properly tuned choke, the power dissipated in the load behind the choke is zero.

Note that the diameter of the insert aperture $(44 \mathrm{~mm})$ is chosen such that its cutoff lies above the dipole mode frequency to avoid its propagation through this aperture.

The dissipated power in a thin wall of the cylindrical insert with a $\mathrm{Cu}$ coating (see Fig. 10) is less than $17 \mathrm{~W}$, corresponding to a power density of $0.08 \mathrm{~W} / \mathrm{cm}^{2}$. This is sufficiently low to cool the insert with liquid nitrogen. The insert of Fig. 11 with a superconducting notch filter has a very low dissipated power and can be cooled with liquid helium.

The results of dipole and monopole HOM simulations are shown in Fig. 13 and Table IV. These results were calculated for a given external quality factor of $Q_{\text {ext }}=$ $\omega_{0} U / P=4.3 \times 10^{5}$ for the fundamental mode. This allows $100 \mathrm{~kW}$ of rf power to be transmitted to a beam of 26-mA current and accelerate it to an energy of $3.7 \mathrm{MeV}$. The maximal electric field on-axis is $20 \mathrm{MV} / \mathrm{m}\left(E_{\text {acc }} \cong\right.$ $10 \mathrm{MV} / \mathrm{m}$ ), which corresponds to a stored rf energy of $U=5.23 \mathrm{~J}$. The dissipated $\mathrm{rf}$ power is found to be only $4.3 \mathrm{~W}$. The total dissipated power taking into account the TE mode [first term of Eq. (32)] is $7 \mathrm{~W}$. Continuous-wave operations are possible with this system.

The dramatic improvement of the threshold current with the suggested HOM damping is illustrated in Table IV. These data demonstrate the effect of HOM damping with the described insert. The threshold currents of untrapped (propagating) modes become large on some orders of magnitude. The 2699 mode is trapped and unstable, but it can be suppressed (see Table III) by TE focusing at $B_{\mathrm{TE}}=0.1 \mathrm{~T}$.

Note also that 1873-, 2699-, and 4466-MHz modes having an unloaded quality of about $4 \times 10^{9}$ are trapped in the first two cells $(1 / 2+1$ - cathode cell and first accelerating cell) and almost have no rf field in the other two cells (see Fig. 14). As a result, they do not couple to the cavity pipe load. These modes have an external quality of approximately $10^{8}$ only due to power losses in the photocathode copper stem cooled by liquid nitrogen. The stem with a $10-\mathrm{mm}$ diameter is positioned into the larger $12-\mathrm{mm}$ pipe hole and attached to the center of the back wall of the cathode cell. There is a coax line formed by these parts. The mentioned three modes penetrate it on decay lengths of $5.7,5.8$, and $6.5 \mathrm{~mm}$, respectively. 

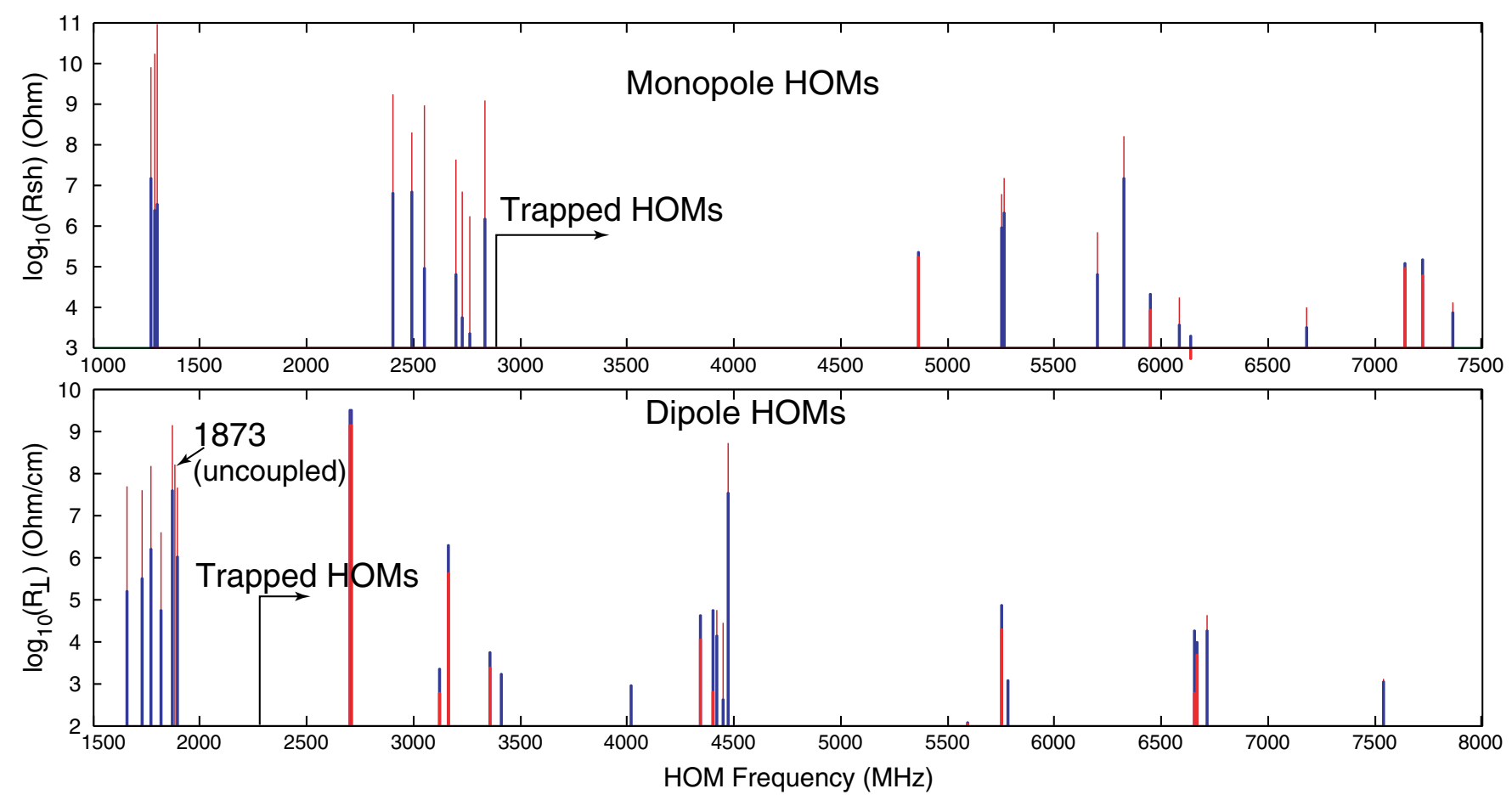

FIG. 13. Monopole [14] and dipole spectra in the rf gun cavity: red-without the insert (see Fig. 5); blue-with inserts shown in Figs. 10 and 11. The cutoff frequencies are marked as "trapped HOMs."

To increase the coupling of these modes with the pipe load and in turn decrease its external quality, we may change slightly the shape of the first accelerating cell. The shaping should not change the fundamental mode frequency and amplitudes in any of the cells. The 4466 mode, for example, is reshaped by slight inclinations (as in Fig. 5) of 2 degrees and an increase of the equator radius by $0.48 \mathrm{~mm}$. The result is $Q_{\text {ext }}=2 \times 10^{5}$ (see Fig. 14). Here, the frequency of the main mode

TABLE IV. Threshold currents $[\mathrm{mA}]$ at $E_{\mathrm{acc}}=25 \mathrm{MV} / \mathrm{m}$ with and without the insert. No TE focusing.

\begin{tabular}{lccccc}
\hline \hline & \multicolumn{3}{c}{ Without insert } & \multicolumn{2}{c}{ With insert } \\
$N$ & $F[\mathrm{MHz}]$ & $I_{\text {th }}, \mathrm{A}$ & $Q_{\text {ext }}$ & $I_{\text {th }}, \mathrm{A}$ & $Q_{\text {ext }}$ \\
\hline 1 & 1653 & & $6.20 \times 10^{7}$ & & $1.95 \times 10^{5}$ \\
2 & 1724 & 1.1 & $1.77 \times 10^{7}$ & 144 & $1.32 \times 10^{5}$ \\
3 & 1766 & & $7.71 \times 10^{6}$ & & $7.97 \times 10^{4}$ \\
4 & 1813 & & $9.72 \times 10^{5}$ & & $1.45 \times 10^{4}$ \\
5 & 1864 & & $4.65 \times 10^{8}$ & & $1.39 \times 10^{7}$ \\
6 & 1873 & & $1.83 \times 10^{8}$ & & $2.00 \times 10^{8}$ \\
7 & 1887 & 3.1 & $8.06 \times 10^{7}$ & 135 & $1.85 \times 10^{6}$ \\
12 & 2699 & 27.3 & $4.76 \times 10^{7}$ & $29^{\mathrm{a}}$ & $4.47 \times 10^{7}$ \\
20 & 3159 & & $2.91 \times 10^{5}$ & & $3.70 \times 10^{6}$ \\
37 & 4466 & & $1.03 \times 10^{8}$ & & $1.03 \times 10^{8}$ \\
89 & 6709 & & $2.83 \times 10^{4}$ & & $2.43 \times 10^{4}$ \\
\hline \hline
\end{tabular}

${ }^{\mathrm{a}}$ This mod is suppressed by $\mathrm{TE}$ focusing at $B_{\mathrm{TE}}=0.1 \mathrm{~T}$ (see Table III). and the field amplitudes in all of the cells remain unchanged.

Finally, note that the external quality of the main mode can be matched to the beam power $P$ as $Q_{\text {ext }} \propto E_{\text {acc }}^{2} / P$ by varying the extent of the coupler into the cavity pipe. Numerical calculations predict that the quality factors of propagating HOMs (with $N=1-5,7$ in Table IV) are changed as $Q_{\mathrm{ext}-N} \sim Q_{\mathrm{ext}}^{1 / 2}$.

Ultimately, when more than just a few $\mathrm{mA}$ are to be accelerated, the use of an rf gun with less than 3.5 cells might be preferable to limit the required rf power

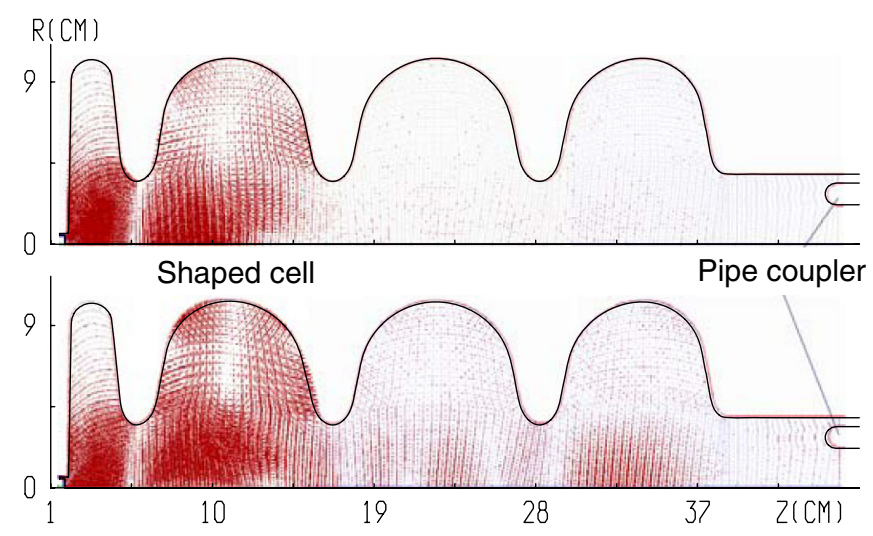

FIG. 14. Electric field distribution of the 4466-MHz mode in the initial cavity (upper picture) and after the shaping of the 1st cell (CLANS2 output is used). 
$(<100 \mathrm{~kW})$ and to reduce the number of trapped modes driving instabilities.

\section{SUMMARY}

Expressions for the coupling impedances of dipole HOMs as a function of the beam current have been derived. The analysis of BBU instabilities in $3 \frac{1}{2}$-cell SRF guns revealed several dipole-driven instabilities, and their associated threshold currents were calculated. In particular, trapped dipole HOMs can be dangerous. A scheme for efficient damping of dipole HOMs by an external load is proposed, resulting in a significant increase of the instability threshold currents. The suppression of the BBU instability of trapped modes by TE focusing is demonstrated, and the possibility of equal TE focusing in all cavity cells is analyzed. New designs of HOM damping consistent with TE mode focusing are suggested. Thus, we believe that the use of SC cavities with less than 3.5 cells for high-current injectors is feasible.

\section{ACKNOWLEDGMENTS}

The authors thank their colleagues from HelmholtzZentrum Berlin for their interest in this work, fruitful discussions, and the editing of this paper. The authors are also thankful to V. Petrov from BINP in Novosibirsk for his help in the clarification of the beam-HOM interaction processes by equivalent electric circuits.
[1] G. R. Neil et al., Phys. Rev. Lett. 84, 662 (2000).

[2] I. Ben-Zvi et al., Nucl. Instrum. Methods Phys. Res., Sect. A 557, 28 (2006).

[3] A. Neumann et al., in Proceedings of the 25th Linear Accelerator Conference, Tsukuba, Japan, 2010, pp. $998-1000$.

[4] V. Volkov, Phys. Rev. ST Accel. Beams 12, 011301 (2009).

[5] A. Arnold et al., Nucl. Instrum. Methods Phys. Res., Sect. A 593, 57 (2008).

[6] V. Volkov, J. Knobloch, and A. Matveenko, Phys. Rev. ST Accel. Beams 13, 084201 (2010).

[7] L. Merminga et al., in Proceedings of the Particle Accelerator Conference, Chicago, IL, 2001 (IEEE, New York, 2001), pp. 173-175.

[8] D. G. Myakishev and V. P. Yakovlev, in Proceedings of the 18th Particle Accelerator Conference, New York, 1999 (IEEE, New York, 1999), pp. 2775-2777.

[9] T. Wangler, $R F$ Linear Accelerators (Wiley-VCH, New York, 2008).

[10] V. Volkov, K. Floettmann, and D. Janssen, in Proceedings of the 2007 Particle Accelerator Conference, Albuquerque, New Mexico (IEEE, New York, 2007).

[11] V. Volkov and D. Janssen, Phys. Rev. ST Accel. Beams 11, 061302 (2008).

[12] V. Volkov et al., in Proceedings of the 9th European Particle Accelerator Conference, Lucerne, 2004 (EPSAG, Lucerne, 2004), pp. 752-754.

[13] A. Arnold (private communication).

[14] V. Volkov and D. Janssen, in Proceedings of the 29th Free Electron Laser Conference, Novosibirsk, Russia, 2007, pp. 228-231. 